\title{
Efficient Testing Combining Design of Experiment and Learn-to-Fly Strategies
}

\author{
Patrick C. Murphy \\ Jay M. Brandon \\ NASA Langley Research Center
}

AIAA SciTech 2017

Atmospheric Flight Mechanics Conference

Grapevine, TX

January 09-13, 2017 


\section{Outline}

- Introduction

- Seeking greater efficiency \& performance through experiment design

- Efficiency gained by collecting the "right amount" of data

- Performance gained by adding statistical rigor

- System Identification Process in Wind Tunnel

- Design of Experiment (DOE)

- Learn-to-Fly (L2F)

- Blended DOE-L2F

- First time testing blended concept - strawman approach

- Work in progress

- Analysis, Results, and Validation Tests

- DOE Tests

- L2F Tests

- Blended DOE-L2F Tests

- Concluding Remarks 


\section{Motivation: Seek Efficiency Using Experiment Design}

- Wide spectrum of modeling demands

- Fidelity requirements

- Aircraft complexity

- Aircraft complexity drive up costs

- Conventional practice in LaRC 12-foot Wind Tunnel (static test)

- $100 \mathrm{~Hz}$ sample rate, dwell for 10 seconds, average data

- 2 data $\mathrm{pts} / \mathrm{min}$

- Simple factorial test for L-59

- 9-Factors: $\alpha, \beta$, and 7 control surfaces

- $2^{9}=512$ test points $=>4.26$ hours

- Reasonable data density often requires $5^{9}=>16,276$ hours ( 8years)!

- Investigators must tradeoff of cost vs fidelity/complexity

- Define purpose of model and required fidelity. What is allowable error?

- Asking for "best possible answer" is not adequate

- Speeding up the modeling process helps anywhere on spectrum 


\section{Aircraft System Identification Process}

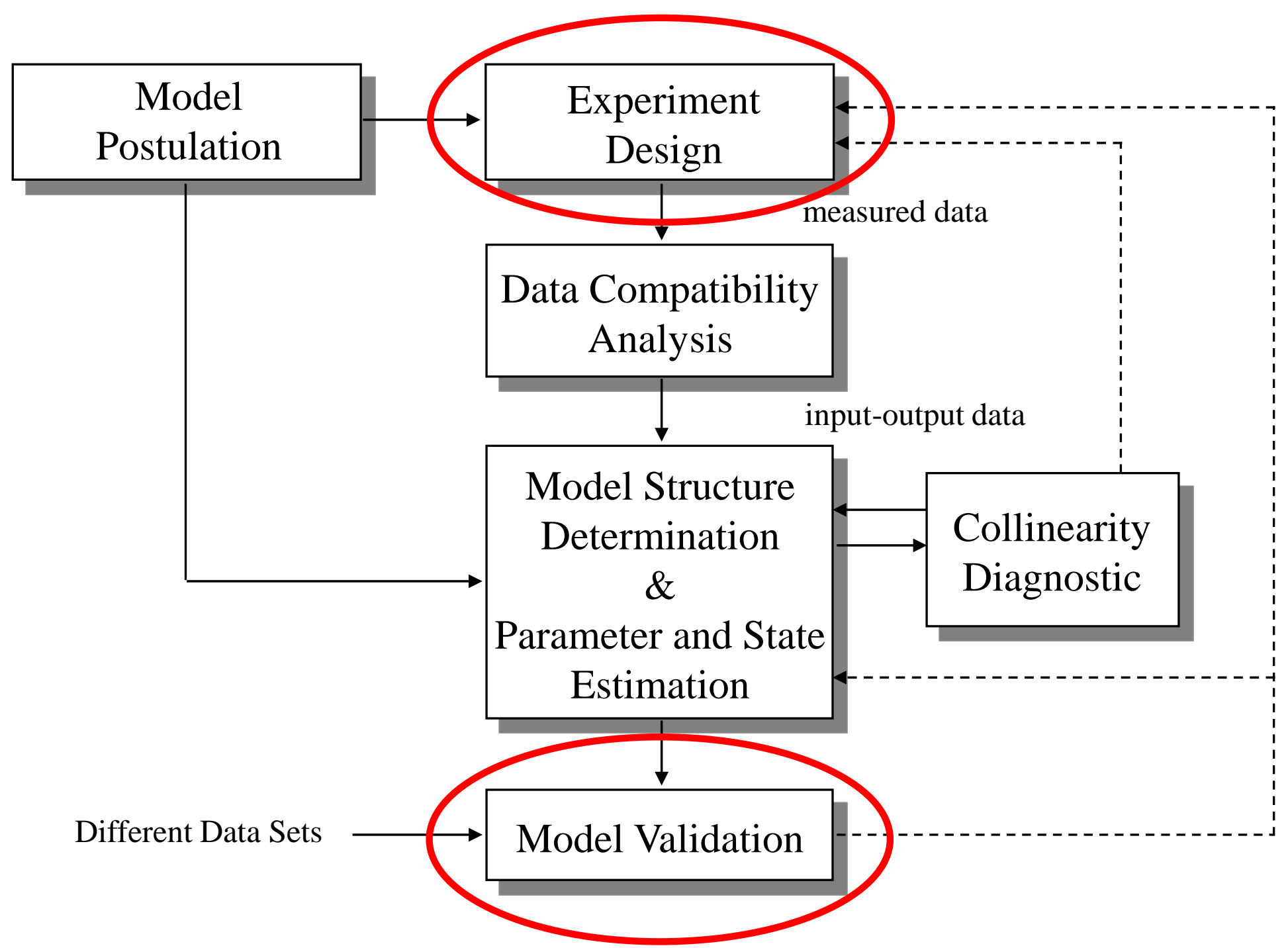




\section{Test vehicle for Wind Tunnel Static Test}

\begin{tabular}{|l|l|c|l|l|l|}
\hline No. & Label & Description & Low & High & Units \\
\hline & & & Value & Value & \\
\hline 1 & aoa & Aircraft alpha & -2 & 20 & deg \\
\hline 2 & beta & Aircraft beta & -5 & 5 & deg \\
\hline 3 & dela_L & Aileron left wing & -25 & 25 & deg \\
\hline 4 & dela_R & Aileron right wing & -25 & 25 & deg \\
\hline 5 & delf_L & Flap left wing & 0 & 40 & deg \\
\hline 6 & delf_R & Flap right wing & 0 & 40 & deg \\
\hline 7 & delr & Rudder & -30 & 30 & deg \\
\hline 8 & dele_L & Elevator left wing & -30 & 30 & deg \\
\hline 9 & dele_R & Elevator right wing & -30 & 30 & deg \\
\hline
\end{tabular}

$>$ L-59 Albatros

$>$ Czech military trainer

$>$ Low-cost off-the-shelf kit

$>12.5 \%$ scale model

$>$ Sport application, RC actuators

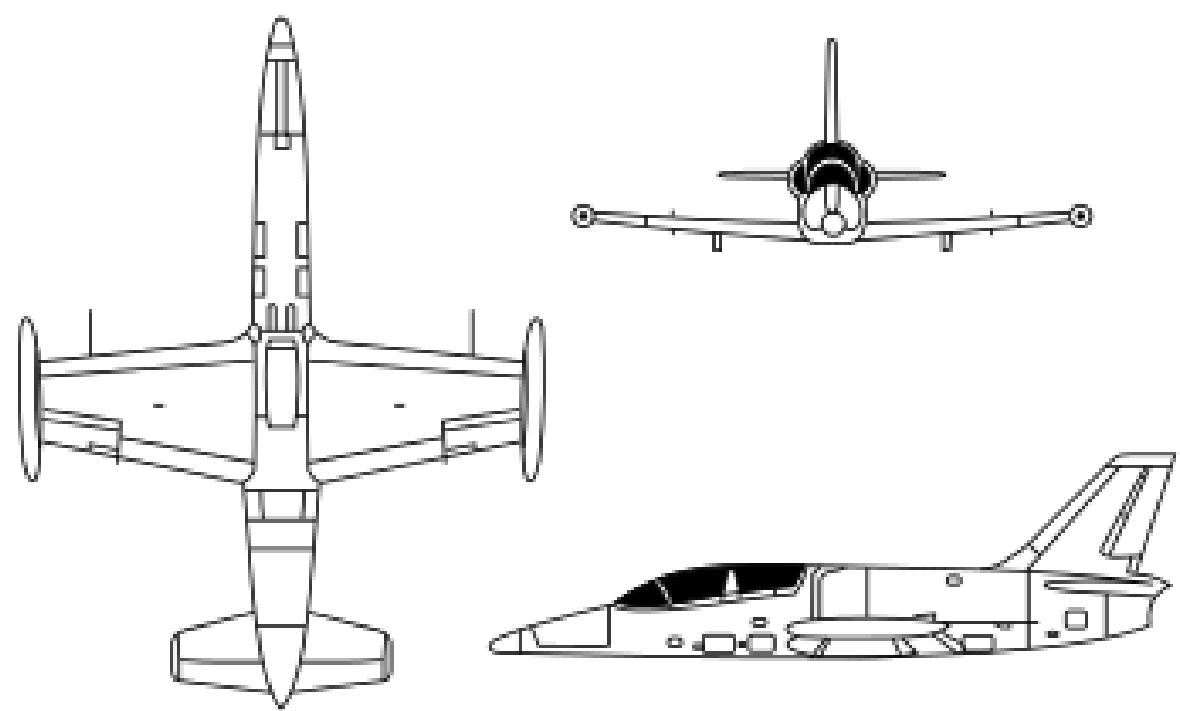




\section{Tenets of Design of Experiment (DOE)}

\section{Sequential testing proceeds only as model complexity requires}

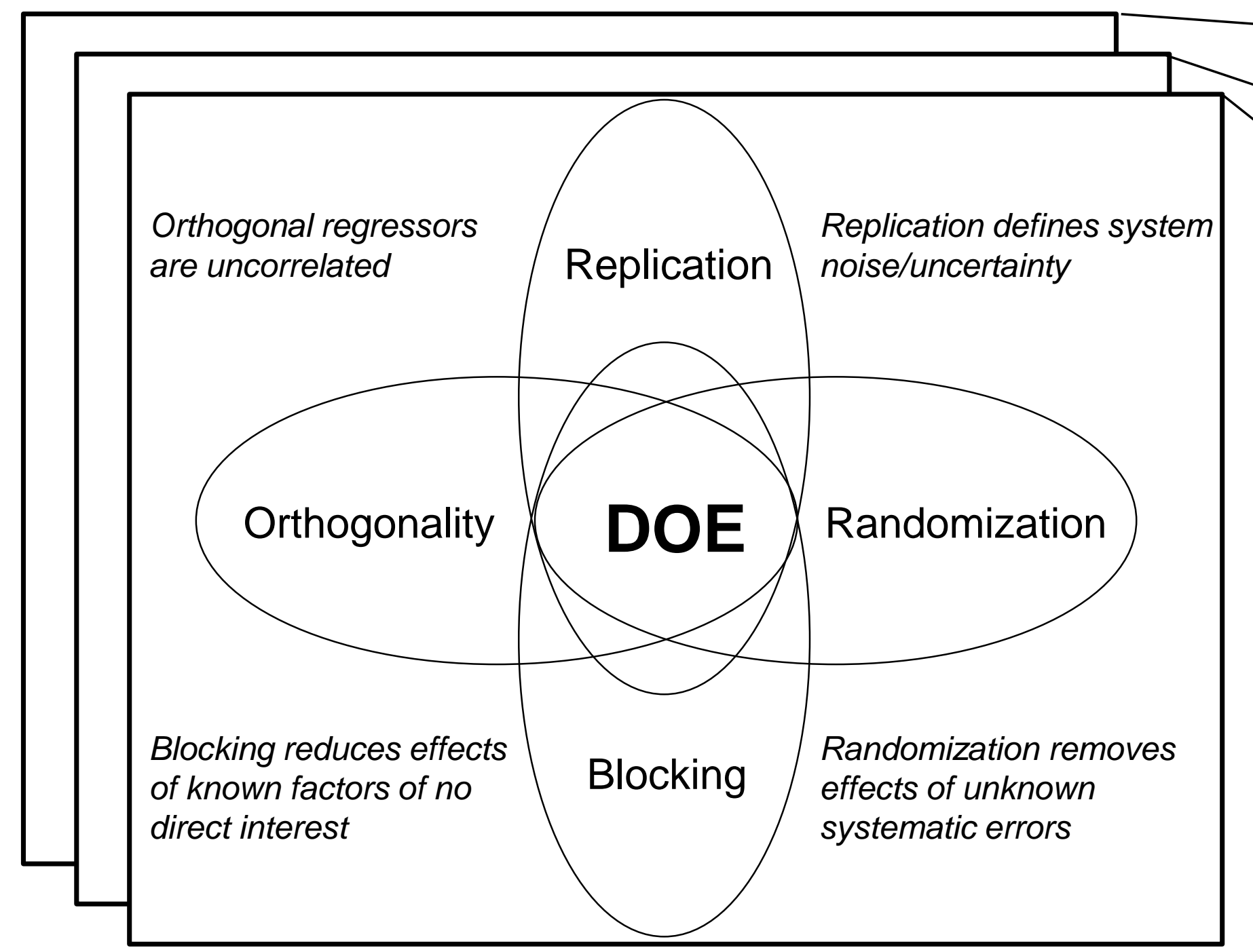

Blocks of data, collected as needed, each followed by model validation 


\section{Block Designs \& Supported Models}

- Full factorial design

$y=B_{0}+\sum_{i} B_{i} x_{i}+\sum \sum_{i \neq j} B_{i j} x_{i} x_{j}+\varepsilon \quad i=1,2, \ldots, k$

- Face-centered design (FCD)

$y=B_{0}+\sum_{i} B_{i} x_{i}+\sum_{i} B_{i i} x_{i}^{2}+\sum_{i \neq j} B_{i j} x_{i} x_{j}+\varepsilon \quad i=1,2, \ldots, k$
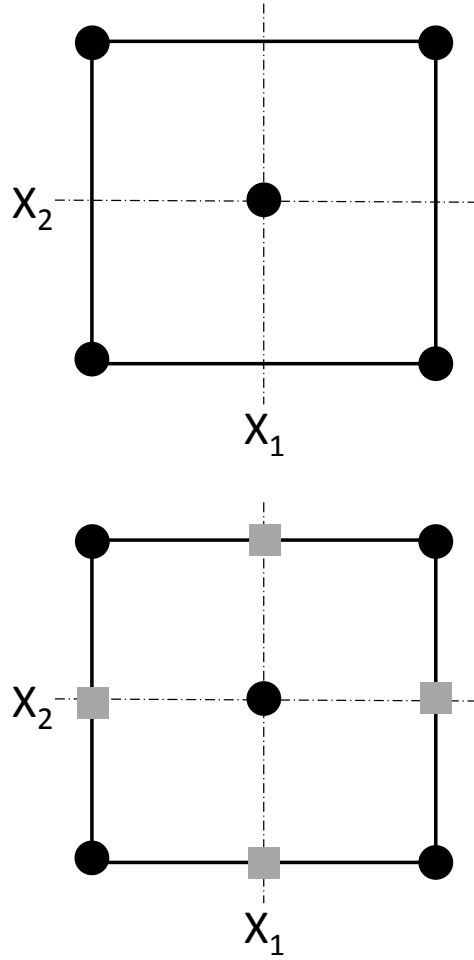

- Nested face-centered design

$y=B_{0}+\sum_{i} B_{i} x_{i}+\sum_{i} B_{i i} x_{i}^{2}+\sum_{i \neq j} B_{i j} x_{i} x_{j}+\sum_{i} B_{i i i} x_{i}^{3}+\varepsilon \quad i=1,2, \ldots, k$

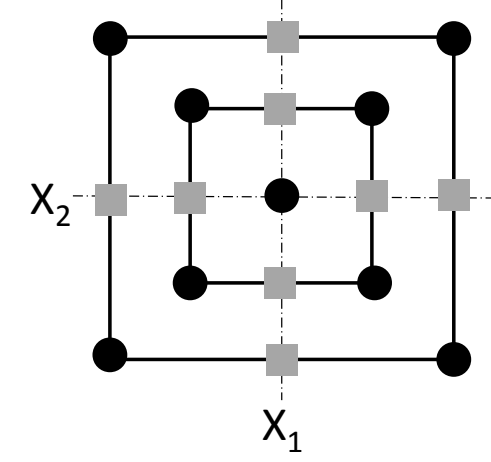




\section{Block 1, DOE Design Metrics (9-factors)}

\begin{tabular}{|l|l|c|c|c|c|}
\hline Block Type & Blocks & Runs & Design Terms & VIF & \% Power \\
\hline & (inclusive) & & & & $2 \sigma, \mathrm{s} / \mathrm{n}=2$ \\
\hline $1 / 4$ Fraction FCD & 1 & 156 & Quadratic & 9.64 & 84.4 \\
\hline
\end{tabular}

Maximum Variance Inflation Factor (VIF), reflects lack of orthogonality in design; desire $\leq 10$

$\%$ Power reflects statistical power of design, manages type- 2 error; desire $\geq 80$

$y=B_{0}+\sum_{i} B_{i} x_{i}+\sum_{i} B_{i i} x_{i}^{2}+\sum_{i \neq j} B_{i j} x_{i} x_{j}+\varepsilon \quad i=1,2, \ldots, k$

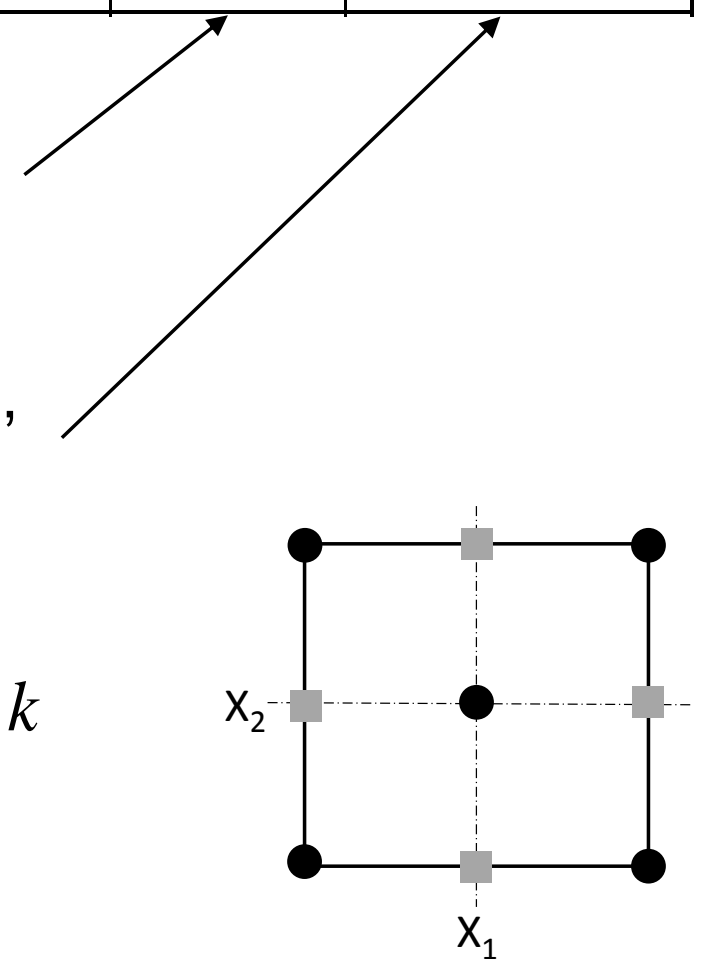

Validation Test Performed after each block of data 


\section{Block 2 added to create Nested FCD}

\begin{tabular}{|l|l|c|c|c|c|}
\hline Block Type & Blocks & Runs & Design Terms & VIF & \% Power \\
\hline & (inclusive) & & & & $2 \sigma, \mathrm{s} / \mathrm{n}=2$ \\
\hline $1 / 4$ Fraction FCD & 1 & 156 & Quadratic & 9.64 & 84.4 \\
\hline Nested FCD & 1,2 & 312 & Quadratic & 22.41 & 86.8 \\
\hline
\end{tabular}

$$
y=B_{0}+\sum_{i} B_{i} x_{i}+\sum_{i} B_{i i} x_{i}^{2}+\sum_{i \neq j} B_{i j} x_{i} x_{j}+\sum_{i} B_{i i i} x_{i}^{3}+\varepsilon \quad i=1,2, \ldots, k
$$

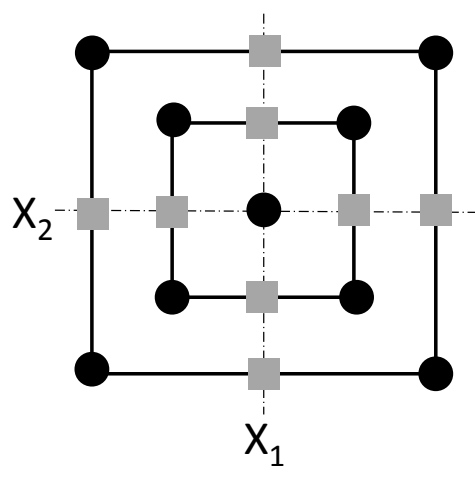

Require optimized design points to reduce VIF 


\section{Final DOE Design, 3-blocks}

\begin{tabular}{|l|l|c|c|c|c|}
\hline Block Type & Blocks & Runs & Design Terms & VIF & $\%$ Power \\
\hline & (inclusive) & & & & $2 \sigma, \mathrm{s} / \mathrm{n}=2$ \\
\hline 1/4 Fraction FCD & 1 & 156 & Quadratic & 9.64 & 84.4 \\
\hline Nested FCD & 1,2 & 312 & Quadratic & 22.41 & 86.8 \\
\hline I-optimal & $1,2,3$ & 366 & Quadratic & 4.0 & 99.9 \\
\hline
\end{tabular}

I-optimal block provides test points that minimize prediction error

Validation Test Performed after each block of data 


\section{DOE Design for 3 blocks}

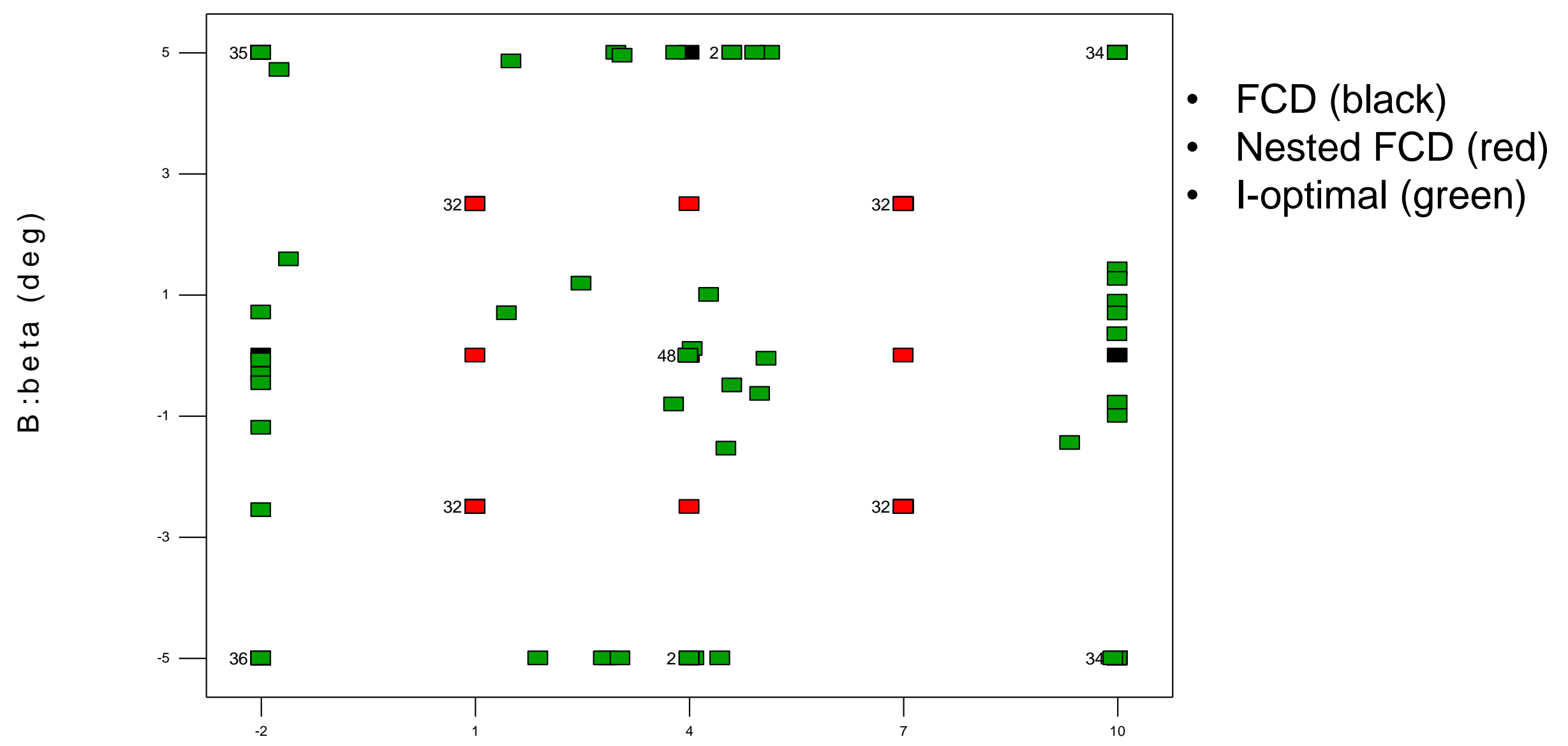

A:aoa 


\section{Stepwise Regression Modeling}

- Stepwise regression used to select model parameters

$$
y=\beta_{0}+\sum_{i} \beta_{i} x_{i}+\sum_{i} \beta_{i i} x_{i}^{2}+\sum \sum_{i \neq j} \beta_{i j} x_{i} x_{j}+\sum_{i} \beta_{i i t} x_{i}^{3}+\ldots+\varepsilon \quad i=1,2, \ldots, 23
$$

- Primary metrics utilized for model selection:

- Stepwise Regression significance level: 95\% - 99\%

- Standard ANOVA table analysis

- Lack of Fit (LOF) measure of model error relative to pure error

- Standard deviation (fit error)

- PRESS (prediction error sum of squares)

- Coefficient of Variation (C.V.\% = std. dev. / mean)

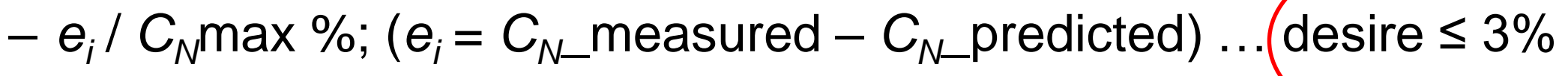

- $\mathrm{R}^{2}$, adjusted $\mathrm{R}^{2}$, predicted $\mathrm{R}^{2}$, (family of metrics)

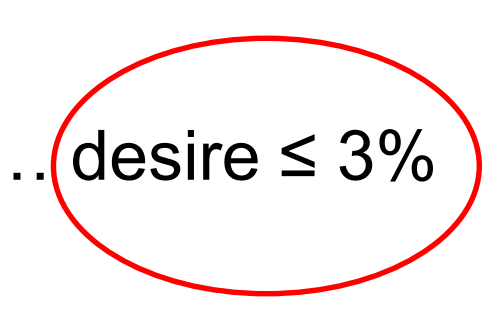

$$
R^{2}=\frac{\text { variation explained }}{\text { total variation }} ; \quad 0<R^{2}<1
$$




\section{Learn-to-Fly (L2F) Testing}

- L2F approach adapted to wind tunnel

- General L2F approach is real-time global modeling of aerodynamics

- Applicable to wind tunnel or flight testing

- Continuous sampling during dynamic test

- This study is a "quasi-static" test

- Continuous sampling while sweeping target points slowly

- Batch processing, stepwise regression

- Key to efficiency: Wide-band orthogonal inputs

- Higher bandwidth (HBW) inputs applied to control surfaces

- Lower bandwidth (LBW) inputs apply to other factors

- L2F experiment design

- Test grid is setup for LBW factors

- LBW trajectories form a nested "FCD-like" design 


\section{Learn-to-Fly (L2F) Trajectories}

Color points by

Standard Order

750

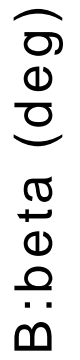

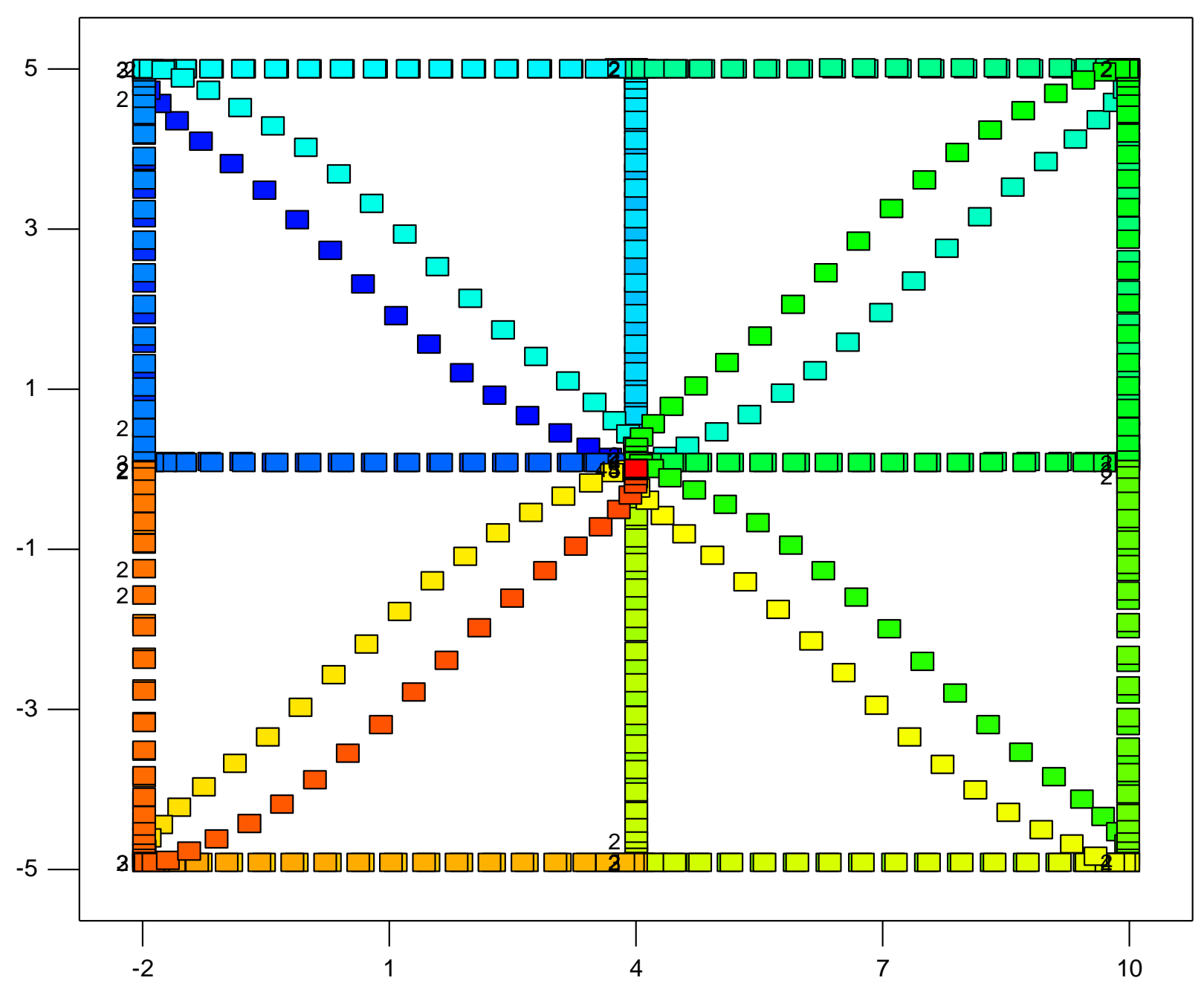

A:aoa (deg) 


\section{Blended DOE-L2F Testing ("quasi-static" test)}

- Use key "efficiency features" of both approaches

- DOE: 4 tenets, sequential testing blocks of data, with validation tests

- L2F: HBW design for factors that accept wide-band inputs

- Blended design both simplifies and complicates final design

- Simplifies 9-LBW experiment to a 2-LBW + 7-HBW experiment

- Complicates evaluation of design metrics

- Strawman blended design

- Design for 9-LBW experiment ensure all factors are included in design

- Keep statistical advantages and design metrics of DOE

- Assume "extra" data between target points enhances modeling

- Assume blended design is obtained by removing redundant $\alpha-\beta$ targets

- Blended designs require rig move slow enough to allow full sweep of controls at each $\alpha-\beta$ target point 


\section{Blended DOE-L2F Design Metrics (9-factors)}

\begin{tabular}{|l|l|c|c|c|c|}
\hline Block Type & Blocks & Runs & Terms & VIF & $\%$ Power \\
\hline & included & target points & & & $2 \sigma, \mathrm{s} / \mathrm{n}=2$ \\
\hline Factorial & 1 & 134 & Linear + 2FI & $1^{*}$ & 99.7 \\
\hline FCD & 1,2 & 156 & Quadratic & 9.68 & 84.2 \\
\hline Nested FCD & $1,2,3$ & 312 & Quadratic & 22.47 & 86.7 \\
\hline I-optimal & $1,2,3,4$ & 384 & Quadratic & 4.85 & 99.9 \\
\hline
\end{tabular}

*Squared factors are aliased

Some Lessons Learned:

Fewer blocks required with continuous sampling

Divide optimal blocks!

$4^{\text {th }}$ block provided too much data for the blended design. 


\section{Blended DOE-L2F Trajectories}

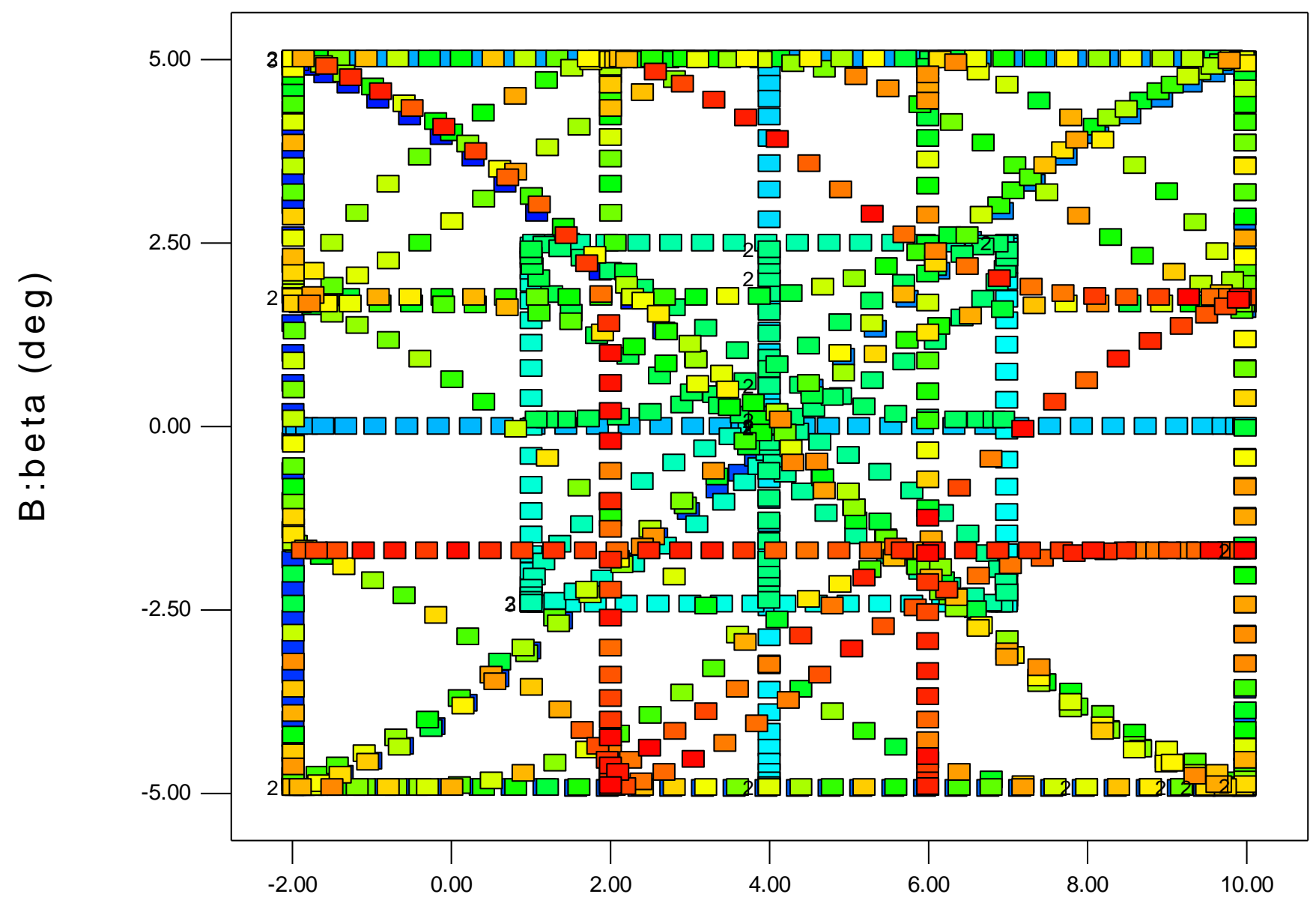

Color points by Standard Order

A:aoa (deg) 


\section{DOE Model (3 blocks)}

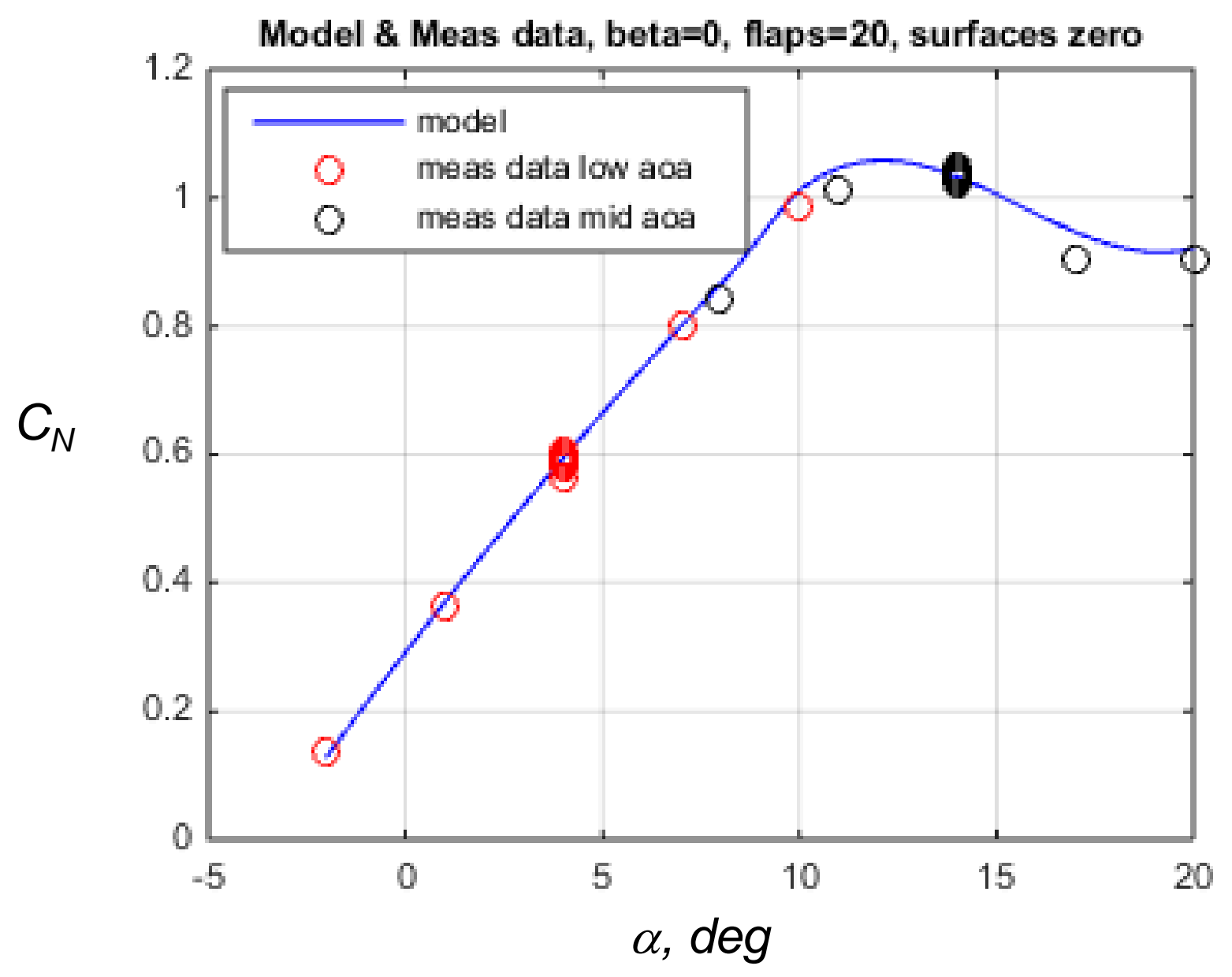




\section{DOE Modeling Progression}

- Block 1 (FCD)

- $1^{\text {st }}$ in series of sequential tests

- Case \#2 - error budget satisfied

- Case \#3 - best model is cubic

- Case \#4 - minimum PRESS

- Case \#6 - minimum Std. Dev

\section{PRESS, Block 1}

0.2

0.175

0.15

0.125

0.1

0.075

0.05

0.025

0

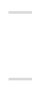

(a)

\section{然}

然

(n)

然




\section{Validation Test, DOE Block 1}

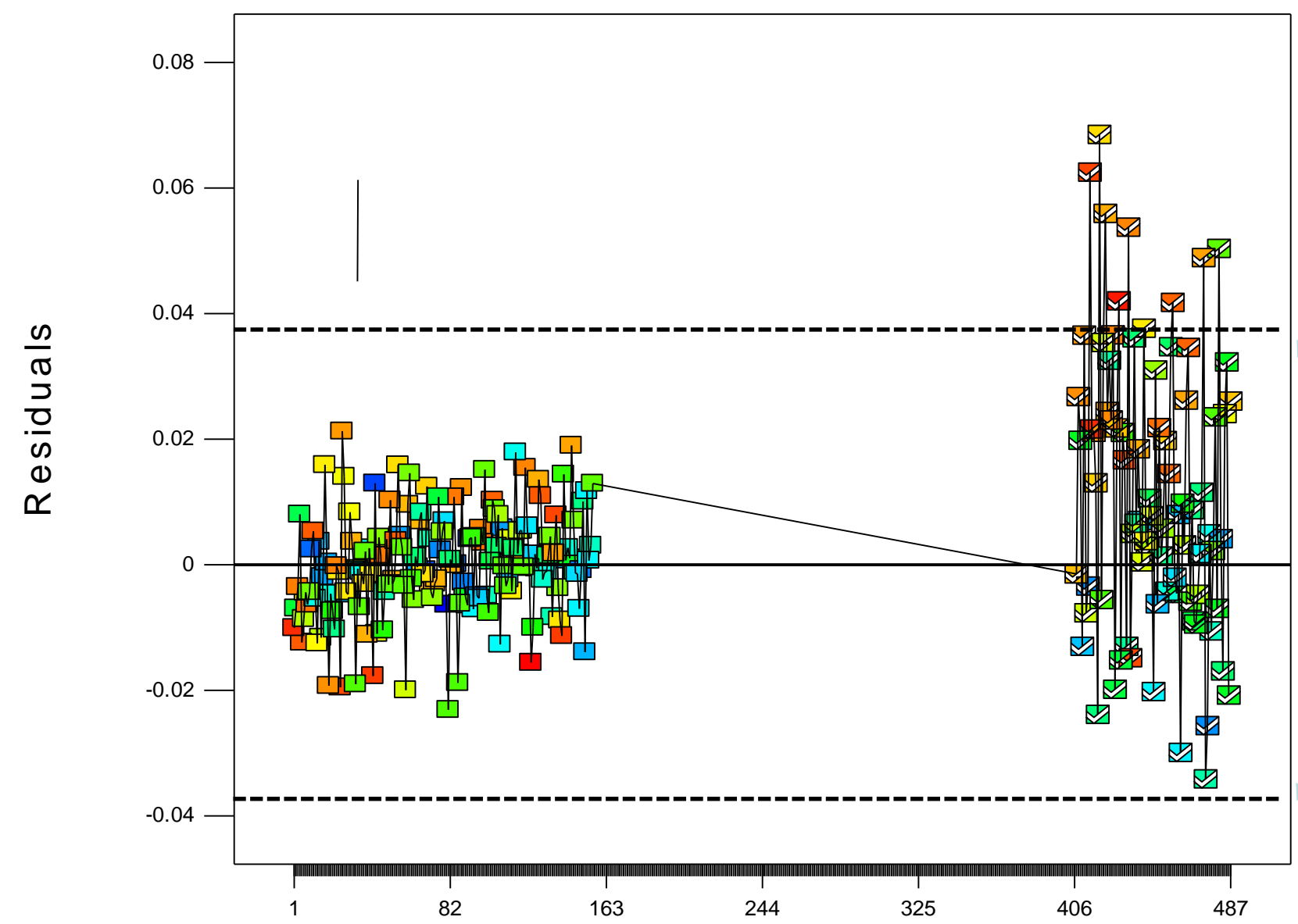

- Residuals vs Run

- Block 1, 1/4 FCD

- $C_{N}$ low $\alpha$ range

- Case \#3 model

- 8 fail $3 \%$ error

$\pm 3 \%$ error

$\pm 3 \%$ error test

- 8 points failed

- 9 of 81 allowed

Run Number

Validation tests reveal true prediction \& bias errors 


\section{Validation Test, DOE Blocks 1-3}

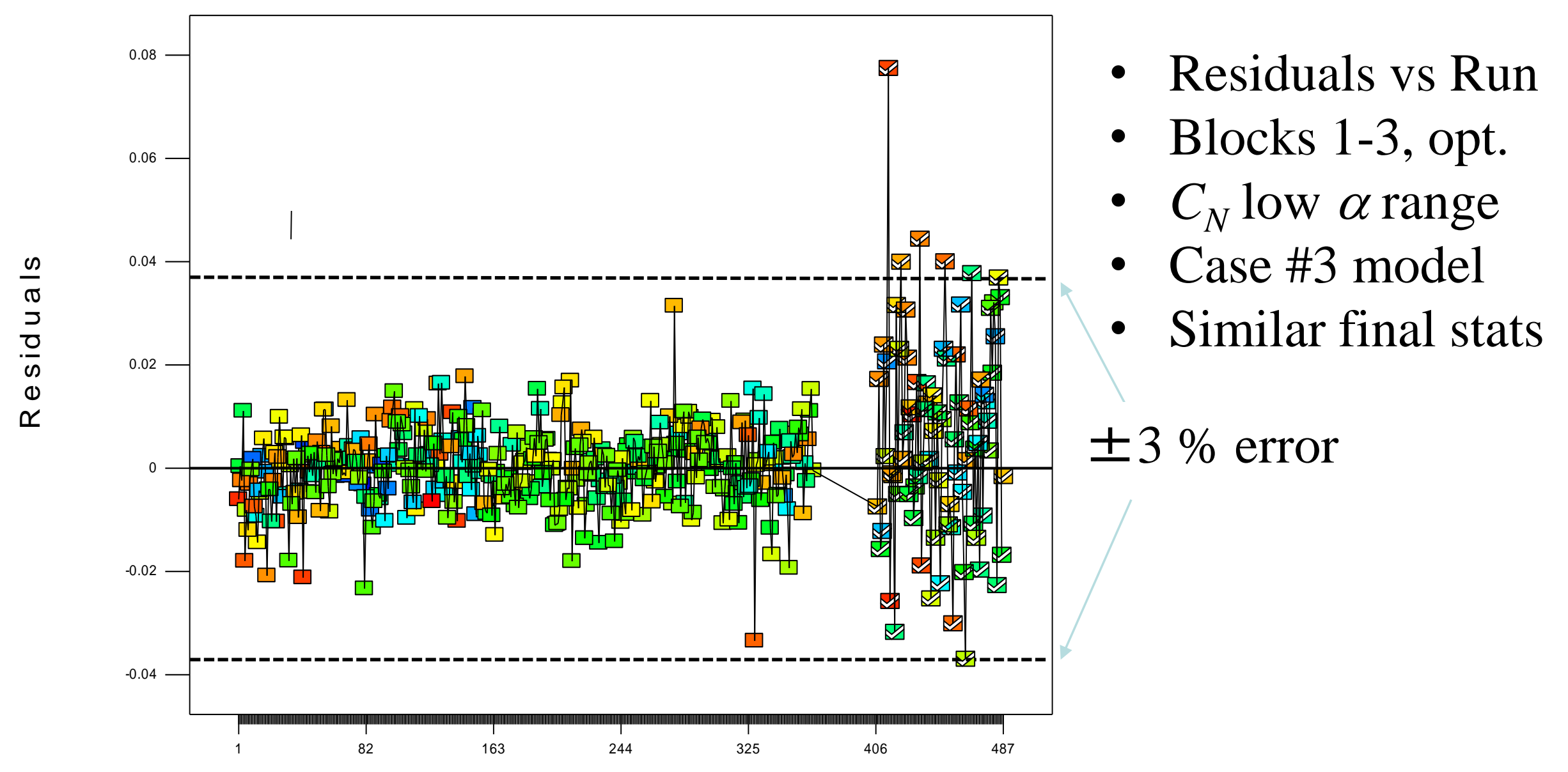

Run Number

Model confirmed by validation test; 6 points fail 3\% error test 


\section{Source of Cubic Terms (DOE blocks 1-3)}

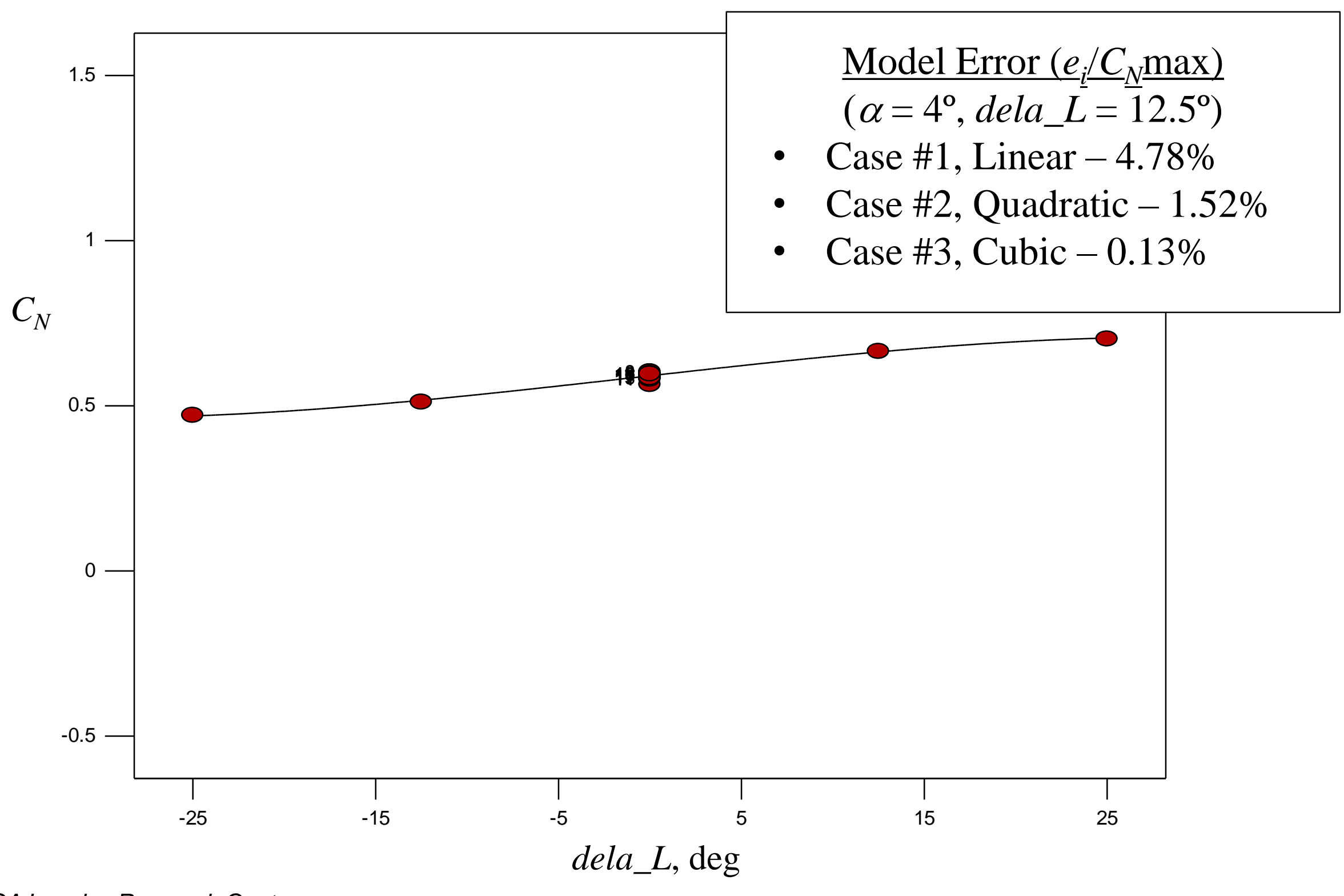




\section{L2F Test in LaRC 12-Foot Tunnel}

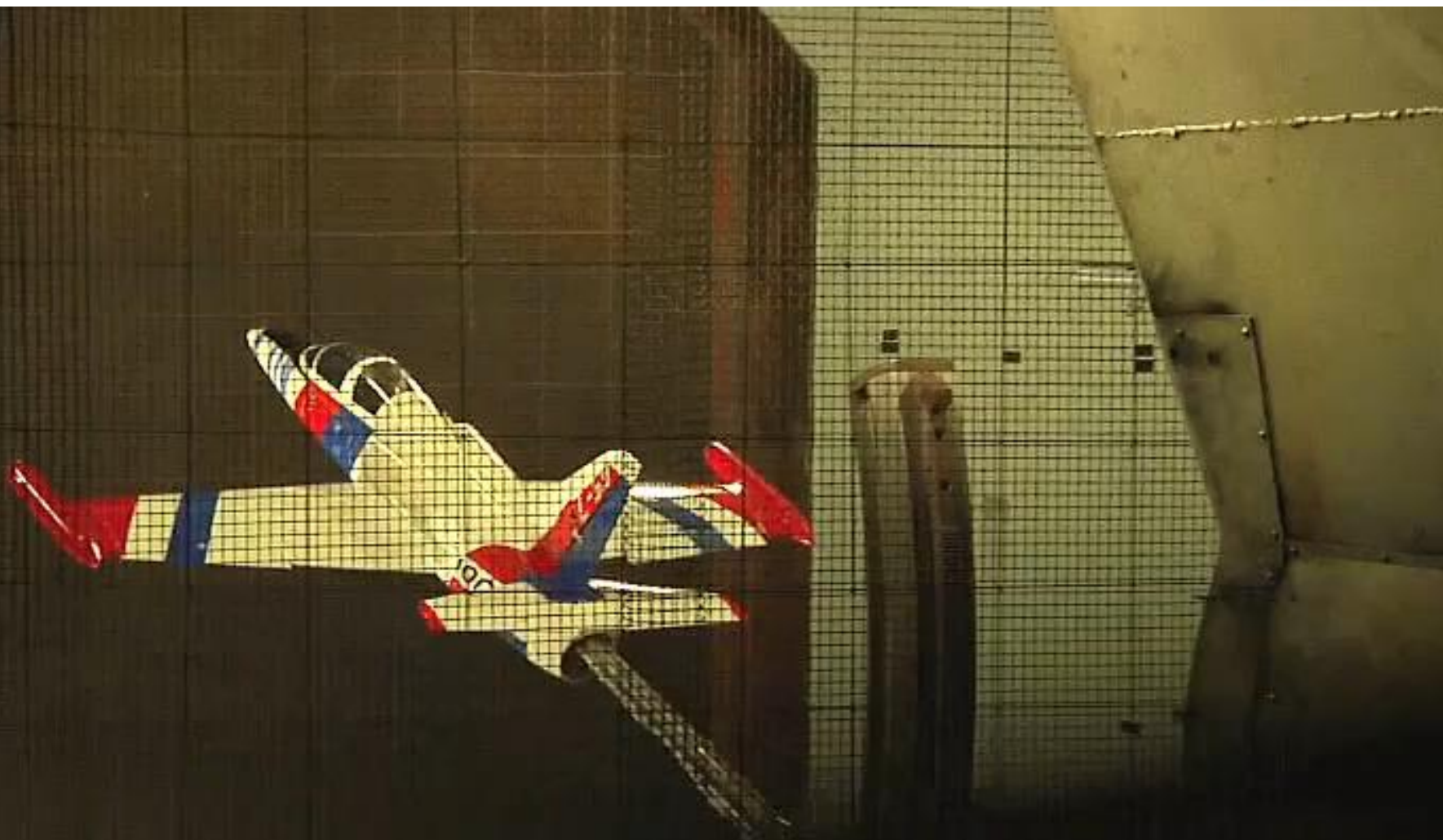




\section{Validation Test, L2F}

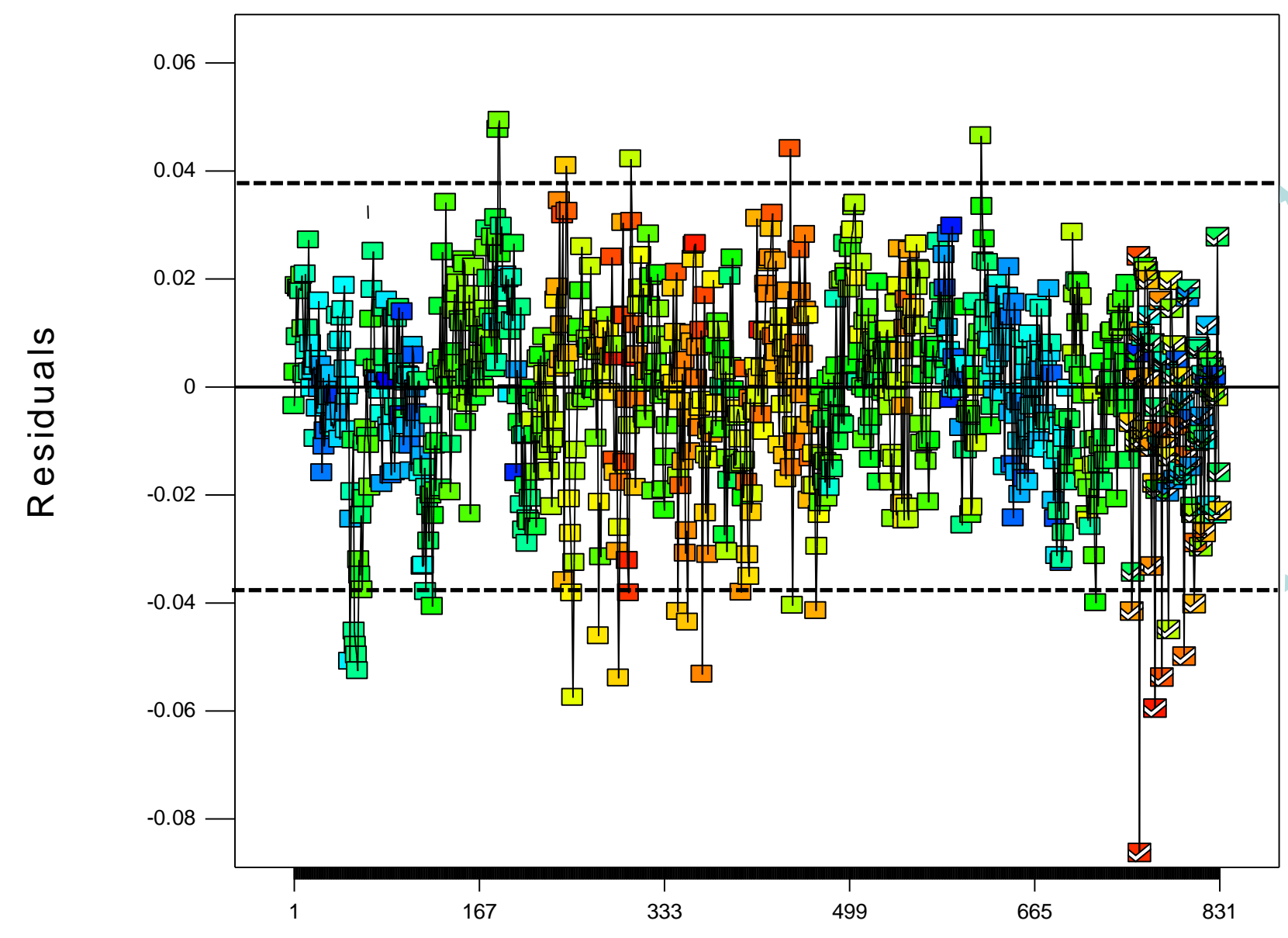

- Residuals vs Run

- Block L2F

- $C_{N}$ low $\alpha$ range

- Case \#3 model

- Similar final stats

$\pm 3 \%$ error

Run Number

Model confirmed by validation test; 7 points fail 3\% error test 


\section{Validation Test, Blended DOE-L2F}

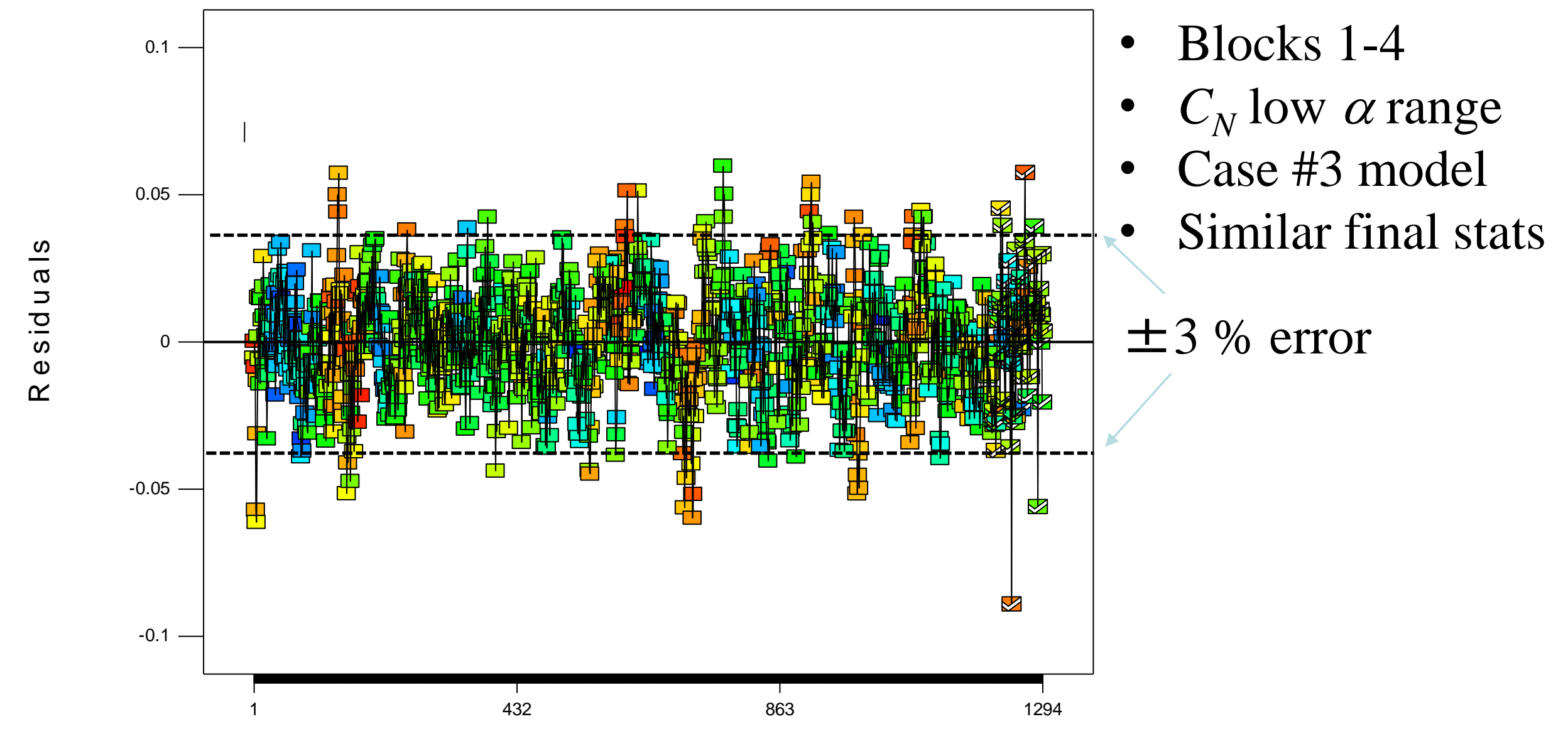

Run Number

Model confirmed by validation test; 6 points fail 3\% error test 


\section{Concluding Remarks}

- Sequential testing \& validation recommended

- Obtain data sequentially as required

- Apply validation test after each block of data

- Efficient test methods demonstrated

- DOE \& L2F approaches provide methods to increase efficiency

- Blending DOE-L2F

- Currently a "work in progress" but shows promise

- Presents a challenge in design phase to combine LBW+HBW factors

- Future Test Refinements

- Fewer blocks required with continuous sampling

- Smaller optimal blocks

- Lower sample rates for "quasi-static" tests

- For "quasi-static" case, lower bandwidth of HBW inputs

- Design must reflect significant data added by HBW factors 


\section{Questions?}

- Contact Information

- patrick.c.murphy@nasa.gov

- 757-864-4071

- jay.m.brandon@nasa.gov

- 757-864-1142
"All models are wrong, but some are useful" George E. P. Box 